\title{
Survival Analysis of Women Exposed to Humidifier Disinfectants during the Perinatal Period: A Nested Case-Control Study
}

\section{Jun Sung Cho}

National Medical Center

Seoheui Choi ( $\nabla$ gabriella77@naver.com )

Hallym University School of Medicine, Dongtan Sacred Heart Hospital

\section{Research Article}

Keywords: humidifier, disinfectant, pregnancy, fetus, survival

Posted Date: November 2nd, 2021

DOl: https://doi.org/10.21203/rs.3.rs-927074/v1

License: (c) (1) This work is licensed under a Creative Commons Attribution 4.0 International License.

Read Full License 


\section{Abstract \\ Background}

Because humidifier disinfectants (HDs) have been prohibited by the government because of their potential serious effects on human body, several studies on the relationship between HD and lung diseases have been separately performed on children and adults; however, to our knowledge, no studies have investigated the effects of HD exposure on pregnant women and their fetuses. Therefore, the present study aimed to investigate the effects of HD exposure on the fetuses of women who were exposed to HDs during pregnancy.

\section{Methods}

We collected data from the Korea Environment Industry \& Technology Institute (KEITI) to investigate the effects of HD exposure on the fetus during pregnancy. Among the applicants, mothers with HD exposure during pregnancy were recruited to determine the damage from HD. Others were included by self-referral or by their guardians. From 2017 to 2020, there were 56 cases. All had been exposed to HDs from 1994 to 2006. Seven cases were excluded because HD exposure did not coincide with pregnancy. We determined the effects of HD exposure on maternal-fetal survival and classified the duration of exposure as it related to the various stages of pregnancy, including prepregnancy, trimester of pregnancy, and seasonal variation. A subgroup analysis based on maternal-fetal survival was performed as a nested case-control study.

\section{Results}

Of the 49 mothers, 22 (44.9\%) survived, and 27 (55.1\%) died. In the group of survivors, the total duration of exposure $(P=0.004)$, the duration of exposure until symptom onset $(P=0.023)$, the duration of exposure before delivery $(P=0.023)$, and duration of exposure before pregnancy $(P=0.010)$ were shorter than those in the group who died. Of the mothers who survived, $50 \%$ were exposed before pregnancy, and $66.7 \%$ of the mothers who died were exposed before delivery.

\section{Conclusions}

A shorter period of HD exposure was associated with a higher likelihood of maternal survival. Also, a shorter time from childbirth to symptom onset was associated with a higher likelihood of maternal-fetal survival.

\section{Background}


Humidifier disinfectants (HDs) have been used in Korea since 1994 to suppress the propagation of microorganisms in humidifiers and to avoid washing the humidifier according to the standards for maintaining their cleanliness while in use [1]. However, since 2006, HDs have been found to contribute to the progressively increasing incidence interstitial lung disease (ILD) of unknown origin, also referred to as HD lung injury (HDLI) [2-5]. In 2011, the Government of the Republic of Korea banned the use of HDs and has established an "HDLI Investigation and Decision Committee" to clinically evaluate registered patients who were presumed to have developed lung injury due to HD use and to determine whether the injury was clinically associated with HD use. Victims of HD use (adults and children or their guardians) voluntarily applied for information on the determination of damage due to HDs, and the Korea Centers for Disease Control and Prevention continued retrospective epidemiological investigations [6, 7].

According to epidemiological studies, $75.6 \%$ of patients with HDLI used humidifiers, and $31.1 \%$ of the users were children [8]. Importantly, $63.5 \%$ of all HDLI cases were among preschool children less than 6 years of age who participated in HD-related damage assessment and $77.1 \%$ of the HDLI cases were infants under 3 years of age [9]. Korea has four distinct seasons, and humidifier usage is most frequent in winter and spring $[10,11]$. Before the realization of their harm to human body, HDs were used frequently to maintain the cleanliness of the humidifier and protect children, especially those with allergies, who suffer from respiratory infections more frequently than adults and pregnant women [8]. According to one study in Korea, the frequency of humidifier use in winter was $45.5 \%$ [10].

In pregnant women, among whom the frequency of HD use was relatively high, there was a need to confirm the effects of in utero fetal exposure to HDs as well as the effects of HD exposure on the mother. Accordingly, the "HD Extrapulmonary Damage Determination Committee" was formed in 2017, and the effects of HDs on pregnant women were determined.

Several studies on the relationship between HD use and lung diseases have been carried out by separating children from adults, but there have been no reports on the effects of HD use on pregnant women and their fetuses. Therefore, this study was conducted to investigate the effects of HDs on the fetuses of women who used HDs during pregnancy.

\section{Methods}

\section{Study participants}

We collected data from the Korea Environment Industry \& Technology Institute (KEITI) to investigate the effects of HD exposure on the fetuses of women who were exposed to HDs during pregnancy from 2017 to 2020. Among the applicants, mothers with HD exposure during pregnancy were recruited to determine the damage from HD. Other participants were included by self-referral or by their guardians. To evaluate the effects of HDs on the fetus, study participants were recruited by two methods. First, an automatic sorting group was formed by chart review to recruit mothers who were exposed to HDs during pregnancy among applicants for damage determination by HDs filed with the KEITI. Second, a volunteer group 
comprising guardians who volunteered for the assessment of HD damage was formed. During pregnancy, mothers with exposure to HDs or fathers of the children were voluntarily recruited by submitting documents containing the patient's medical records and informed consent from guardians to the KEITI. HD use during pregnancy was confirmed by submission of a receipt for the use of HDs and actual photos of the HDs in use at the time of medical record submission. A total of 56 applicants who were recruited in the present study were classified as patients who were evaluated for the determination of external pulmonary damage due to HD use, and all medical records were reviewed retrospectively.

The study protocol was approved by the Institutional Review Board of Hallym University Dongtan Sacred Heart Hospital (IRB No. 2019-12-014-001), and written informed consent to participate in the study was obtained from the parents or their guardians at enrollment.

\section{Collection of disinfectant information}

The data obtained from the medical records included the following maternal data: date of birth, start and end dates of HD use, survival status, date of death (if relevant), starting date of the mother's symptoms, estimated due date, and fetal height and weight at the time of the visit to the obstetrician. If these data were missing from the medical records at the prepregnancy visit, the other medical records were checked to assess HD-related damage. The following fetal data were collected: date of birth, gestational age, birth weight, and survival status within 28 days of life. All data were retrospectively investigated through the medical records. Hospitalization records or clinical records those obtained from clinics were used to determine the date of symptom onset for the mother.

\section{Measurement of data}

The age of the mother was defined as the age at symptom onset. Body mass index (BMI) of the mother was calculated using the following formula: $\mathrm{BMI}=$ body weight $/$ height ${ }^{2}\left(\mathrm{~kg} / \mathrm{m}^{2}\right)$. Total duration of HD exposure in the mother was defined as the period from the first date of HD use to the end of HD use or the date of maternal death. The period from childbirth to the onset of symptoms in the mother was expressed as a negative value in cases where the symptoms were observed before birth. The period from pregnancy until when symptoms became apparent was calculated from the start date of symptoms to the estimated date of pregnancy, and the period from the start of HD use until when symptoms became apparent was calculated. In cases where HD use was initiated preconception, the duration of HD exposure was calculated from the estimated date of conception to the end of HD use or until childbirth.

For fetuses, with the exception of cases with estimated dates of conception, the duration of HD exposure was defined as the number of weeks of pregnancy in which the fetus was exposed to HDs. If HDs were used before pregnancy, the duration of HD exposure was calculated from the start of pregnancy. If HDs were used before pregnancy, the exposure period before pregnancy was converted to weeks. Gestational age and birth weight of the neonate were reported as blank in cases of fetal demise or miscarriage. Estimated pregnancy start date (month) was identified and classified as spring (March to May), summer (June to August), autumn (September to November), and winter (December to February). The start date of HD use was classified as prepregnancy, first trimester (1-12 weeks), second trimester (13-26 weeks), 
and third trimester (27-40 weeks). The study subjects were further subdivided according to HD use into those who started HD use from before pregnancy to the first trimester and those who started HD use after the first trimester.

\section{Data analysis}

The chi-square test was used to compare categorical data, and Student's $t$ test was used to compare numerical data. Differences between groups with estimated pregnancy dates in summer and nonsummer seasons were compared. The effect of HD exposure in early pregnancy was determined by comparing subjects based on the trimester in which HD use was initiated. All statistical analyses were performed using SPSS version 21.0 (SPSS Inc., Chicago, IL, USA).

\section{Results}

\section{Clinical characteristics of the participants}

The present study cohort included patients with overlapping HD use periods and pregnancy periods. Among a total of 56 fetal cases (42 cases in 2017, 12 cases in 2018, and 2 cases in 2019) filed by the $\mathrm{KEITI}$, the present study included 49 cases after the exclusion of 7 cases, including 2 cases where pregnancy and childbirth occurred before HD use and 5 cases where pregnancy and childbirth occurred after the end of HD use (Figure 1).

\section{Duration of HD use and maternal mortality}

Of the 49 mothers, 22 survived (44.9\%) and 27 died (55.1\%). The maternal survival analysis showed that the total duration of HD use ( $642.4 \pm 470.1$ vs. $1019.5 \pm 917.3$ days, $p=0.004)$, the duration of HD use until the onset of symptoms ( $553.0 \pm 417.1$ vs. $799.0 \pm 663.4$ days, $p=0.023)$, the duration of HD use before birth ( $376.3 \pm 314.4$ vs. $646.1 \pm 546.1$ days, $p=0.023)$, and the duration of HD use before pregnancy ( $22.3 \pm 36.9$ vs. $56.6 \pm 71.4$ weeks, $p=0.010$ ) were shorter in the mothers who survived than in those who died. The analysis of HD use start date categorized according to the specific trimester revealed that $50 \%$ of the mothers who survived used HDs before pregnancy and that $66.7 \%$ of the mothers who died used HDs before birth. None of the mothers started using HDs in the first or third trimester of pregnancy (Table 1). 
Table 1

Comparison of demographic features of groups based on maternal outcome

\begin{tabular}{|c|c|c|c|}
\hline & $\begin{array}{l}\text { Maternal } \\
\text { survival } \\
(n=22)\end{array}$ & $\begin{array}{l}\text { Maternal death } \\
(n=27)\end{array}$ & $\begin{array}{l}p \\
\text { value }\end{array}$ \\
\hline Maternal age (years) & $32.1 \pm 3.2$ & $31.5 \pm 3.3$ & 0.686 \\
\hline Maternal height (cm) & $158.2 \pm 5.9$ & $161.6 \pm 5.1$ & 0.877 \\
\hline Maternal weight (kg) & $59.3 \pm 11.8$ & $61.2 \pm 14.3$ & 0.621 \\
\hline Maternal BMI $\left(\mathrm{kg} / \mathrm{m}^{2}\right)$ & $23.6 \pm 3.6$ & $23.3 \pm 4.8$ & 0.379 \\
\hline Neonatal survival (\%) & $15 / 22(68.2)$ & $17 / 27(63.0)$ & 0.703 \\
\hline Total HD use duration (days) & $642.4 \pm 470.1$ & $1019.5 \pm 917.3$ & 0.004 \\
\hline Duration from birth to symptom onset (weeks) & $24.2 \pm 46.1$ & $23.0 \pm 54.5$ & 0.459 \\
\hline $\begin{array}{l}\text { Duration from conception to symptom onset } \\
\text { (weeks) }\end{array}$ & $38.0 \pm 18.6$ & $37.2 \pm 6.8$ & 0.074 \\
\hline $\begin{array}{l}\text { Duration from start of HD use to symptom onset } \\
\text { (days) }\end{array}$ & $553.0 \pm 417.1$ & $799.0 \pm 663.4$ & 0.023 \\
\hline Duration of intrauterine exposure (days) & $185.7 \pm 93.8$ & $237.1 \pm 75.7$ & 0.276 \\
\hline Duration from start of HD use to birth (days) & $376.3 \pm 314.4$ & $646.1 \pm 546.1$ & 0.023 \\
\hline \multicolumn{4}{|l|}{ Duration of HD exposure when started } \\
\hline during pregnancy (weeks) & $10.1 \pm 11.9$ & $6.9 \pm 10.3$ & 0.579 \\
\hline before conception (weeks) & $22.3 \pm 36.9$ & $56.6 \pm 71.4$ & 0.010 \\
\hline Gestational age (weeks) & $34.1 \pm 5.8$ & $32.8 \pm 6.9$ & 0.248 \\
\hline Birth weight (g) & $2372.9 \pm 1072.0$ & $\begin{array}{l}2621.5 \pm \\
1099.3\end{array}$ & 0.742 \\
\hline Estimated conception season (\%) & & & 0.454 \\
\hline Spring (Mar-May) & $5 / 22(22.7)$ & $12 / 27(44.4)$ & \\
\hline Summer (Jun-Aug) & $10 / 22(45.5)$ & $8 / 27(29.6)$ & \\
\hline Autumn (Sep-Nov) & $3 / 22(13.6)$ & $3 / 27(11.1)$ & \\
\hline Winter (Dec-Feb) & $4 / 22(18.2)$ & $4 / 27(14.8)$ & \\
\hline $\begin{array}{l}\text { Classification by season } \\
\text { (Summer vs other seasons) (\%) }\end{array}$ & $10 / 22(45.5)$ & $8 / 27(29.6)$ & 0.253 \\
\hline Estimated trimester of exposure (n, \%) & & & 0.038 \\
\hline
\end{tabular}




\begin{tabular}{|llll|}
\hline & $\begin{array}{l}\text { Maternal } \\
\text { survival } \\
(\mathbf{n = 2 2 )}\end{array}$ & $\begin{array}{l}\text { Maternal death } \\
\mathbf{( n = 2 7 )}\end{array}$ & $\begin{array}{c}\boldsymbol{p} \\
\text { value }\end{array}$ \\
\hline Prenatal exposure & $11 / 22(50)$ & $18 / 27(66.7)$ & \\
\hline 1st trimester (1-12 weeks) & $3 / 22(13.6)$ & $0 / 27(0)$ & \\
\hline 2nd trimester (13-26 weeks) & $5 / 22(22.7)$ & $9 / 27(33.3)$ & \\
\hline 3rd trimester (27-40 weeks) & $3 / 22(13.6)$ & $0 / 27(0)$ & \\
\hline $\begin{array}{l}\text { Classification by trimester } \\
\text { (Prenatal-1st vs 2nd -3rd ) (n, \%) }\end{array}$ & $14 / 22(63.6)$ & $18 / 27(66.7)$ & 0.825 \\
\hline
\end{tabular}

\section{Subgroup analysis of neonatal outcomes according to maternal survival}

Among the 22 surviving mothers, 15 (68.2\%) neonates survived and 7 (31.8\%) neonates died. The duration from birth to the start of clinical symptoms was shorter ( $17.9 \pm 33.6$ vs. $36.8 \pm 65.9$ weeks, $p=$ $0.013)$ and the average gestational age was higher ( $35.5 \pm 4.0$ vs. $28.7 \pm 9.0$ weeks, $p=0.020)$ among the surviving neonates compared with those who died.

Among the 27 mothers who died, 17 (63.0\%) neonates survived and 10 (37.0\%) neonates died. In this group of mothers who died, the duration from birth to the start of clinical symptoms was longer in the neonates who survived than in those who died $(39.2 \pm 61.8$ vs. $-7.6 \pm 3.9$ weeks, $p<0.001)$ (Table 2$)$. 
Table 2

Comparison of demographic features of groups based on maternal outcome with fetal outcome subgroup analysis

\begin{tabular}{|c|c|c|c|c|c|c|}
\hline & \multicolumn{3}{|c|}{ Maternal Survival $(n=22)$} & \multicolumn{3}{|c|}{ Maternal Death $(n=27)$} \\
\hline & $\begin{array}{l}\text { Neonatal } \\
\text { survival }\end{array}$ & $\begin{array}{l}\text { Neonatal } \\
\text { death }\end{array}$ & $\begin{array}{l}p \\
\text { value }\end{array}$ & $\begin{array}{l}\text { Neonatal } \\
\text { survival }\end{array}$ & $\begin{array}{l}\text { Neonatal } \\
\text { death }\end{array}$ & $\begin{array}{l}p \\
\text { value }\end{array}$ \\
\hline & $(n=15)$ & $(n=7)$ & & $(n=17)$ & $(n=10)$ & \\
\hline Maternal age (years) & $31.8 \pm 3.1$ & $32.9 \pm 3.6$ & 0.800 & $31.3 \pm 3.7$ & $32.0 \pm 2.6$ & 0.101 \\
\hline Maternal height (cm) & $157.5 \pm 6.0$ & $\begin{array}{l}159.8 \pm \\
5.8\end{array}$ & 0.620 & $161.3 \pm 5.6$ & $162.4 \pm 3.9$ & 0.249 \\
\hline Maternal weight (kg) & $57.8 \pm 12.1$ & $\begin{array}{l}63.2 \pm \\
11.5\end{array}$ & 0.989 & $60.9 \pm 16.0$ & $61.7 \pm 11.6$ & 0.460 \\
\hline $\begin{array}{l}\text { Maternal BMI } \\
\left(\mathrm{kg} / \mathrm{m}^{2}\right)\end{array}$ & $23.2 \pm 3.8$ & $24.7 \pm 3.3$ & 0.973 & $23.2 \pm 4.7$ & $23.6 \pm 5.7$ & 0.694 \\
\hline Neonatal survival (\%) & $15 / 22(68.2)$ & $7 / 22(31.8)$ & & $17 / 27(63.0)$ & $10 / 27(37.0)$ & \\
\hline $\begin{array}{l}\text { Total HD use } \\
\text { duration (days) }\end{array}$ & $\begin{array}{l}609.7 \pm \\
501.4\end{array}$ & $\begin{array}{l}712.4 \pm \\
422.4\end{array}$ & 0.804 & $\begin{array}{l}1270.4 \pm \\
973.9\end{array}$ & $\begin{array}{l}593.0 \pm \\
650.8\end{array}$ & 0.100 \\
\hline $\begin{array}{l}\text { Duration from birth to } \\
\text { symptom onset } \\
\text { (weeks) }\end{array}$ & $17.9 \pm 33.6$ & $\begin{array}{l}36.8 \pm \\
65.9\end{array}$ & 0.013 & $39.2 \pm 61.8$ & $-7.6 \pm 3.9$ & $<0.001$ \\
\hline $\begin{array}{l}\text { Duration from } \\
\text { conception to } \\
\text { symptom onset } \\
\text { (weeks) }\end{array}$ & $44.4 \pm 15.9$ & $\begin{array}{l}21.9 \pm \\
16.0\end{array}$ & 0.529 & $41.5 \pm 4.1$ & $33.5 \pm 6.6$ & 0.347 \\
\hline $\begin{array}{l}\text { Duration from start } \\
\text { of HD use to } \\
\text { symptom onset } \\
\text { (days) }\end{array}$ & $\begin{array}{l}500.9 \pm \\
401.0\end{array}$ & $\begin{array}{l}657.0 \pm \\
461.2\end{array}$ & 0.377 & $\begin{array}{l}901.2 \pm \\
661.4\end{array}$ & $\begin{array}{l}606.0 \pm \\
660.6\end{array}$ & 0.403 \\
\hline $\begin{array}{l}\text { Duration of } \\
\text { intrauterine exposure } \\
\text { (days) }\end{array}$ & $\begin{array}{l}200.9 \pm \\
98.1\end{array}$ & $\begin{array}{l}153.3 \pm \\
81.1\end{array}$ & 0.148 & $\begin{array}{l}243.6 \pm \\
73.3\end{array}$ & $\begin{array}{l}226.1 \pm \\
82.4\end{array}$ & 0.892 \\
\hline $\begin{array}{l}\text { Duration from start } \\
\text { of HD use to birth }\end{array}$ & $\begin{array}{l}365.7 \pm \\
255.1\end{array}$ & $\begin{array}{l}399.1 \pm \\
439.5\end{array}$ & 0.235 & $\begin{array}{l}627.1 \pm \\
511.1\end{array}$ & $\begin{array}{l}678.4 \pm \\
628.6\end{array}$ & 0.841 \\
\hline \multicolumn{7}{|c|}{ Duration (weeks) of HD exposure when started } \\
\hline during pregnancy & $10.1 \pm 12.6$ & $\begin{array}{l}10.1 \pm \\
11.2\end{array}$ & 0.489 & $8.0 \pm 10.2$ & $5.1 \pm 10.7$ & 0.465 \\
\hline before conception & $18.9 \pm 27.6$ & $\begin{array}{l}29.6 \pm \\
53.9\end{array}$ & 0.232 & $54.2 \pm 65.6$ & $60.7 \pm 83.8$ & 0.867 \\
\hline $\begin{array}{l}\text { Gestational age } \\
\text { (weeks) }\end{array}$ & $35.5 \pm 4.0$ & $28.7 \pm 9.0$ & 0.020 & $37.5 \pm 3.1$ & $25.7 \pm 4.3$ & 0.207 \\
\hline
\end{tabular}




\begin{tabular}{|c|c|c|c|c|c|c|}
\hline \multirow[b]{2}{*}{ Birthweight (g) } & \multicolumn{3}{|c|}{ Maternal Survival $(n=22)$} & \multicolumn{3}{|c|}{ Maternal Death $(n=27)$} \\
\hline & $\begin{array}{l}2578.2 \pm \\
836.8\end{array}$ & $\begin{array}{l}1620.0 \pm \\
1698.9\end{array}$ & 0.031 & $\begin{array}{l}3097.2 \pm \\
616.0\end{array}$ & $\begin{array}{l}837.5 \pm \\
324.3\end{array}$ & 0.324 \\
\hline \multicolumn{3}{|c|}{ Estimated pregnancy season (\%) } & 0.869 & & & 0.411 \\
\hline Spring (Mar-May) & $4 / 15(26.7)$ & 1/7 (14.3) & & $6 / 17(35.3)$ & $6 / 10(60.0)$ & \\
\hline Summer (Jun-Aug) & $6 / 15(40.0)$ & $4 / 7(57.1)$ & & $5 / 17(29.4)$ & $3 / 10(30.0)$ & \\
\hline Autumn (Sep-Nov) & 2/15 (13.3) & $1 / 7(14.3)$ & & $3 / 17(17.6)$ & $0 / 10(0.0)$ & \\
\hline Winter (Dec-Feb) & $3 / 15(20.0)$ & $1 / 7(14.3)$ & & $3 / 17(17.6)$ & $1 / 10(10.0)$ & \\
\hline $\begin{array}{l}\text { Classification by } \\
\text { season } \\
\text { (Summer vs other } \\
\text { seasons) (\%) }\end{array}$ & \multicolumn{5}{|c|}{$\begin{array}{l}\text { (Summer vs other } \\
\text { seasons) }(\%)\end{array}$} & 0.974 \\
\hline \multicolumn{3}{|c|}{$\begin{array}{l}\text { Estimated trimester of exposure (n, } \\
\%)\end{array}$} & 0.550 & & & 0.260 \\
\hline Prenatal exposure & $8 / 15(53.3)$ & $3 / 7(42.9)$ & & $\begin{array}{l}10 / 17 \\
(58.8)\end{array}$ & $8 / 10(20.0)$ & \\
\hline $\begin{array}{l}\text { 1st trimester }(1-12 \\
\text { weeks) }\end{array}$ & $1 / 15(6.7)$ & $2 / 7(28.6)$ & & $0 / 17(0.0)$ & $0 / 10(0.0)$ & \\
\hline $\begin{array}{l}\text { 2nd trimester (13-26 } \\
\text { weeks) }\end{array}$ & $4 / 15(26.7)$ & $1 / 7(14.3)$ & & $7 / 17(41.2)$ & $2 / 10(20.0)$ & \\
\hline $\begin{array}{l}\text { 3rd trimester (27-40 } \\
\text { weeks) }\end{array}$ & 2/15 (13.3) & $3 / 7(42.9)$ & & $0 / 17(58.8)$ & $0 / 10(0.0)$ & \\
\hline $\begin{array}{l}\text { Classification by } \\
\text { trimester } \\
\text { (Prenatal-1st vs } 2 \text { nd } \\
-3 \text { rd })(n, \%)\end{array}$ & $9 / 15(60.0)$ & $5 / 7(71.4)$ & 0.604 & $\begin{array}{l}10 / 17 \\
(58.8)\end{array}$ & $8 / 10(80)$ & 0.260 \\
\hline
\end{tabular}

\section{HD exposure and neonatal death}

Among the 49 mothers, 32 neonates survived (65.3\%) and 17 neonates died (34.6\%). The comparison of these two neonatal groups revealed that, albeit not statistically significant, the total duration of HD use ( $96.07 \pm 845.7$ vs. $642.2 \pm 555.7$ days, $p=0.057$ ) and the duration of HD use until the onset of symptoms $(29.5 \pm 51.4$ vs. $11.9 \pm 47.6$ weeks, $p=0.315)$ tended to be longer and the gestational age $(36.7 \pm 3.6$ vs. $26.4 \pm 5.4$ weeks, $p=0.227$ ) and the birth weight of the neonates tended to be higher (2877.6 \pm 749.0 vs. $1172.9 \pm 1090.7 \mathrm{~g}, p=0.634)$ in the surviving neonates than in those who died (Table 3 ). 
Table 3

Comparison of demographic features of groups based on fetal outcome

\begin{tabular}{|c|c|c|c|}
\hline & $\begin{array}{l}\text { Neonatal } \\
\text { survival } \\
(n=32)\end{array}$ & $\begin{array}{l}\text { Neonatal death } \\
(n=17)\end{array}$ & $\begin{array}{l}p \\
\text { value }\end{array}$ \\
\hline Maternal age (years) & $31.5 \pm 3.4$ & $32.4 \pm 3.0$ & 0.357 \\
\hline Maternal height $(\mathrm{cm})$ & $159.3 \pm 6.0$ & $160.9 \pm 4.9$ & 0.559 \\
\hline Maternal weight $(\mathrm{kg})$ & $59.3 \pm 13.9$ & $62.4 \pm 11.0$ & 0.535 \\
\hline Maternal BMI (kg/m²) & $23.2 \pm 4.2$ & $24.2 \pm 4.3$ & 0.634 \\
\hline Maternal survival (\%) & 15/32(46.9) & $7 / 17(41.2)$ & 0.703 \\
\hline Total HD use duration (days) & $960.7 \pm 845.7$ & $642.2 \pm 555.7$ & 0.057 \\
\hline Duration from birth to symptom onset (weeks) & $29.5 \pm 51.4$ & $11.9 \pm 47.6$ & 0.315 \\
\hline $\begin{array}{l}\text { Duration from conception to symptom onset } \\
\text { (weeks) }\end{array}$ & $43.1 \pm 12.0$ & $29.9 \pm 11.1$ & 0.394 \\
\hline $\begin{array}{l}\text { Duration from start of HD use to symptom onset } \\
\text { (days) }\end{array}$ & $720.4 \pm 586.5$ & $628.3 \pm 564.3$ & 0.392 \\
\hline Duration of intrauterine exposure (days) & $223.6 \pm 87.1$ & $196.1 \pm 87.5$ & 0.750 \\
\hline Duration from start of HD use to birth & $504.6 \pm 426.4$ & $563.4 \pm 561.0$ & 0.628 \\
\hline \multicolumn{4}{|l|}{ Duration (weeks) of HD exposure when started } \\
\hline during pregnancy & $9.0 \pm 11.2$ & $7.2 \pm 10.9$ & 0.574 \\
\hline before conception & $37.6 \pm 53.8$ & $47.9 \pm 72.7$ & 0.604 \\
\hline Gestational age (weeks) & $36.7 \pm 3.6$ & $26.4 \pm 5.4$ & 0.227 \\
\hline Birthweight (g) & $2877.6 \pm 749.0$ & $\begin{array}{l}1172.9 \pm \\
1090.7\end{array}$ & 0.634 \\
\hline Estimated pregnancy season (\%) & & & 0.649 \\
\hline Spring (Mar-May) & 10/32 (31.3) & 7/17 (41.2) & \\
\hline Summer (Jun-Aug) & $11 / 32(34.4)$ & 7/17 (41.2) & \\
\hline Autumn (Sep-Nov) & $5 / 32(15.6)$ & $1 / 17(5.9)$ & \\
\hline Winter (Dec-Feb) & $6 / 32(18.8)$ & 2/17 (11.8) & \\
\hline Classification by season & 11/32 (34.4) & $7 / 17(41.2)$ & 0.638 \\
\hline \multicolumn{4}{|l|}{ (Summer vs other seasons) (\%) } \\
\hline Estimated trimester of exposure (n, \%) & & & 0.461 \\
\hline
\end{tabular}




\begin{tabular}{|llll|}
\hline & $\begin{array}{l}\text { Neonatal } \\
\text { survival } \\
(\mathbf{n = 3 2})\end{array}$ & $\begin{array}{l}\text { Neonatal death } \\
(\mathbf{n = 1 7 )}\end{array}$ & $\begin{array}{l}\boldsymbol{p} \\
\text { value }\end{array}$ \\
\hline Prenatal exposure & $18 / 32(56.3)$ & $11 / 17(64.7)$ & \\
\hline 1st trimester (1-12 weeks) & $1 / 32(3.1)$ & $2 / 17(11.8)$ \\
\hline 2nd trimester (13-26 weeks) & $11 / 32(34.4)$ & $3 / 17(17.6)$ & \\
\hline 3rd trimester (27-40 weeks) & $2 / 32(6.3)$ & $1 / 17(5.9)$ & \\
\hline Classification by trimester & $19 / 32(59.4)$ & $13 / 17(76.5)$ & 0.231 \\
(Prenatal-1st vs 2nd -3rd ) (n, \%) & & & \\
\hline
\end{tabular}

\section{Subgroup analysis of maternal outcomes according to neonatal survival}

Among the 32 surviving neonates, 15 mothers survived (46.9\%), and 17 mothers died (53.1\%). The total duration of HD use $(609.7 \pm 501.4$ vs. $1270.4 \pm 973.9$ days, $p=0.023)$, the duration of HD use from birth to the development of clinical symptoms ( $17.9 \pm 33.6 \mathrm{vs.} 39.2 \pm 61.8$ weeks, $p=0.008)$, the duration of HD use until the onset of symptoms (500.9 \pm 401.0 vs. $901.2 \pm 661.4$ days, $p=0.021)$, the duration of HD use before birth ( $365.7 \pm 255.1$ vs. $627.1 \pm 511.1$ days, $p=0.002)$, and the duration of HD use before pregnancy ( $18.9 \pm 27.6$ vs. $54.2 \pm 65.6$ weeks, $p=0.001)$ were shorter and the birthweight of the neonate was lower ( $2578.2 \pm 836.8$ vs. $3097.2 \pm 616.0 \mathrm{~g}, p=0.046)$ in the mothers who survived than in those who died.

Among the 17 neonates who died, 7 mothers survived (41.2\%) and 10 mothers died (58.8\%). The duration of HD use from birth to the development of clinical symptoms was longer (36.8 \pm 65.9 vs. $-7.6 \pm 3.9$ weeks, $p<0.001$ ), the duration of HD use from conception to the development of clinical symptoms was shorter $(21.9 \pm 16.0$ vs. $33.5 \pm 6.6$ weeks, $p=0.016)$, and the birthweight was higher $(1620.0 \pm 1698.9$ vs. $837.5 \pm 324.3 \mathrm{~g}, p=0.015$ ) in the mothers who survived than in those who died (Table 4). 
Table 4

Comparison of demographic features of groups based on fetal outcome with maternal outcome subgroup analysis

\begin{tabular}{|c|c|c|c|c|c|c|}
\hline & \multicolumn{3}{|c|}{ Neonatal survival $(n=32)$} & \multicolumn{3}{|c|}{ Neonatal death $(n=17)$} \\
\hline & $\begin{array}{l}\text { Maternal } \\
\text { survival }(n= \\
15)\end{array}$ & $\begin{array}{l}\text { Maternal } \\
\text { death }(\mathrm{n}= \\
17)\end{array}$ & $\begin{array}{l}p \\
\text { value }\end{array}$ & $\begin{array}{l}\text { Maternal } \\
\text { survival ( } \mathrm{n} \\
=7 \text { ) }\end{array}$ & $\begin{array}{l}\text { Maternal } \\
\text { death }(n= \\
10)\end{array}$ & $\begin{array}{l}p \\
\text { value }\end{array}$ \\
\hline Maternal age (years) & $31.8 \pm 3.1$ & $31.1 \pm 3.7$ & 0.223 & $32.9 \pm 3.6$ & $32.0 \pm 2.6$ & 0.514 \\
\hline Maternal height (cm) & $157.5 \pm 6.0$ & $161.3 \pm 5.6$ & 0.951 & $\begin{array}{l}159.8 \pm \\
5.8\end{array}$ & $162.4 \pm 3.9$ & 0.690 \\
\hline Maternal weight (kg) & $57.8 \pm 12.1$ & $60.9 \pm 16.0$ & 0.420 & $\begin{array}{l}63.2 \pm \\
11.5\end{array}$ & $61.7 \pm 11.6$ & 0.886 \\
\hline $\begin{array}{l}\text { Maternal BMI } \\
\left(\mathrm{kg} / \mathrm{m}^{2}\right)\end{array}$ & $23.2 \pm 3.8$ & $23.2 \pm 47$ & 0.476 & $24.7 \pm 3.3$ & $23.6 \pm 5.7$ & 0.363 \\
\hline Maternal survival (\%) & $15 / 32(46.9)$ & $17 / 32(53.1)$ & & $7 / 17(41.2)$ & $10 / 17(58.8)$ & \\
\hline $\begin{array}{l}\text { Total duration of HD } \\
\text { use duration (days) }\end{array}$ & $\begin{array}{l}609.7 \pm \\
501.4\end{array}$ & $\begin{array}{l}1270.4 \pm \\
973.9\end{array}$ & 0.023 & $\begin{array}{l}712.4 \pm \\
422.4\end{array}$ & $\begin{array}{l}593.0 \pm \\
650.8\end{array}$ & 0.782 \\
\hline $\begin{array}{l}\text { Duration from birth } \\
\text { to symptom onset } \\
\text { (weeks) }\end{array}$ & $17.9 \pm 33.6$ & $39.2 \pm 61.8$ & 0.008 & $\begin{array}{l}36.8 \pm \\
65.9\end{array}$ & $-7.6 \pm 3.9$ & $<0.001$ \\
\hline $\begin{array}{l}\text { Duration from } \\
\text { conception to } \\
\text { symptom onset } \\
\text { (weeks) }\end{array}$ & $44.4 \pm 15.9$ & $41.5 \pm 4.1$ & 0.282 & $\begin{array}{l}21.9 \pm \\
16.0\end{array}$ & $33.5 \pm 6.6$ & 0.016 \\
\hline $\begin{array}{l}\text { Duration from start } \\
\text { of HD use to } \\
\text { symptom onset } \\
\text { (days) }\end{array}$ & $\begin{array}{l}500.9 \pm \\
401.0\end{array}$ & $\begin{array}{l}901.2 \pm \\
661.4\end{array}$ & 0.021 & $\begin{array}{l}657.0 \pm \\
461.2\end{array}$ & $\begin{array}{l}606.0 \pm \\
660.6\end{array}$ & 0.885 \\
\hline $\begin{array}{l}\text { Duration of } \\
\text { intrauterine exposure } \\
\text { (days) }\end{array}$ & $\begin{array}{l}200.9 \pm \\
98.1\end{array}$ & $\begin{array}{l}243.6 \pm \\
73.3\end{array}$ & 0.073 & $\begin{array}{l}153.3 \pm \\
81.1\end{array}$ & $\begin{array}{l}226.1 \pm \\
82.4\end{array}$ & 0.644 \\
\hline $\begin{array}{l}\text { Duration from start } \\
\text { of HD use to birth } \\
\text { (days) }\end{array}$ & $\begin{array}{l}365.7 \pm \\
255.1\end{array}$ & $\begin{array}{l}627.1 \pm \\
511.1\end{array}$ & 0.002 & $\begin{array}{l}399.1 \pm \\
439.5\end{array}$ & $\begin{array}{l}678.4 \pm \\
628.6\end{array}$ & 0.627 \\
\hline \multicolumn{7}{|c|}{ Duration (weeks) of HD exposure when started } \\
\hline during pregnancy & $10.1 \pm 12.6$ & $8.0 \pm 10.2$ & 0.461 & $\begin{array}{l}10.1 \pm \\
11.2\end{array}$ & $5.1 \pm 10.7$ & 0.850 \\
\hline before conception & $18.9 \pm 27.6$ & $54.2 \pm 65.6$ & 0.001 & $\begin{array}{l}29.6 \pm \\
53.9\end{array}$ & $60.7 \pm 83.8$ & 0.529 \\
\hline $\begin{array}{l}\text { Gestational age } \\
\text { (weeks) }\end{array}$ & $35.5 \pm 4.0$ & $37.5 \pm 3.1$ & 0.104 & $28.7 \pm 9.0$ & $25.7 \pm 4.3$ & 0.056 \\
\hline
\end{tabular}




\begin{tabular}{|c|c|c|c|c|c|c|}
\hline \multirow[b]{2}{*}{ Birthweight (g) } & \multicolumn{3}{|c|}{ Neonatal survival $(n=32)$} & \multicolumn{3}{|c|}{ Neonatal death $(n=17)$} \\
\hline & $\begin{array}{l}2578.2 \pm \\
836.8\end{array}$ & $\begin{array}{l}3097.2 \pm \\
616.0\end{array}$ & 0.046 & $\begin{array}{l}1620.0 \pm \\
1698.9\end{array}$ & $\begin{array}{l}837.5 \pm \\
324.3\end{array}$ & 0.015 \\
\hline \multicolumn{3}{|c|}{ Estimated pregnancy season (\%) } & 0.904 & & & 0.229 \\
\hline Spring (Mar-May) & $4 / 15(26.7)$ & $6 / 17(35.3)$ & & $1 / 7(14.3)$ & $6 / 10(60.0)$ & \\
\hline $\begin{array}{l}\text { Summer } \\
\text { (Jun-Aug) }\end{array}$ & $6 / 15(40.0)$ & $5 / 17(29.4)$ & & $4 / 7(57.1)$ & $3 / 10(30.0)$ & \\
\hline $\begin{array}{l}\text { Autumn } \\
\text { (Sep-Nov) }\end{array}$ & 2/15 (13.3) & $3 / 17(17.6)$ & & $1 / 7(14.3)$ & $0 / 10(0.0)$ & \\
\hline $\begin{array}{l}\text { Winter } \\
\text { (Dec-Feb) }\end{array}$ & $3 / 15(20.0)$ & $3 / 17(17.6)$ & & $1 / 7(14.3)$ & $1 / 10(10.0)$ & \\
\hline $\begin{array}{l}\text { Classification by } \\
\text { season } \\
\text { (Summer vs other } \\
\text { seasons) (\%) }\end{array}$ & $6 / 15(40.0)$ & $5 / 17(29.4)$ & & $4 / 7(57.1)$ & $3 / 10(30.0)$ & \\
\hline $\begin{array}{l}\text { Estimated trimester of } \\
\%)\end{array}$ & xposure (n, & & 0.269 & & & 0.155 \\
\hline Prenatal exposure & $8 / 15(53.3)$ & $\begin{array}{l}10 / 17 \\
(58.8)\end{array}$ & & $3 / 7(42.9)$ & $8 / 10(80.0)$ & \\
\hline $\begin{array}{l}1 \text { st trimester (1-12 } \\
\text { weeks) }\end{array}$ & $1 / 15(6.7)$ & $0 / 17(0.0)$ & & $2 / 7(28.6)$ & $0 / 10(0.0)$ & \\
\hline $\begin{array}{l}\text { 2nd trimester (13-26 } \\
\text { weeks) }\end{array}$ & $4 / 15(26.7)$ & $7 / 17(41.2)$ & & $1 / 7(14.3)$ & $2 / 10(20.0)$ & \\
\hline $\begin{array}{l}\text { 3rd trimester (27-40 } \\
\text { weeks) }\end{array}$ & 2/15 (13.3) & $0 / 17(0.0)$ & & $1 / 7(14.3)$ & $0 / 10(0.0)$ & \\
\hline $\begin{array}{l}\text { Classification by } \\
\text { trimester } \\
\text { (Prenatal-1st vs } 2 \text { nd } \\
-3 r d)(n, \%)\end{array}$ & $9 / 15(60.0)$ & $\begin{array}{l}10 / 17 \\
(58.8)\end{array}$ & 0.946 & $5 / 7(71.4)$ & $8 / 10(80.0)$ & 0.682 \\
\hline
\end{tabular}

\section{Discussion}

According to the data from the 2009-2011 period by the Korea Statistical Office, in 2012, the incidence of infant deaths under 1 year of age was 3.0 per 1000 live births, the maternal mortality rate was 17.2 per 100,000 live births, and the perinatal mortality rate was 3.1 per 1000 live births for neonates who were born over 28 weeks' gestational age and who were less than 7 days old [12]. These data encompassing 
the present study period included 1677 deaths among 7498 victims of ILD caused by HD exposure, with a mortality rate of $22.4 \%$ [13]. In the present study, 17 neonates (14.3\%) and 27 mothers (55.1\%) died among 49 mothers who used HDs during pregnancy. The observed high mortality rate among the pregnant women in the present study may be attributed to specific immunological changes during pregnancy. There are several effects of HDs on the fetus during pregnancy: 1) increase in maternal blood concentration of the medication which can directly affect the fetus given that the medication can pass through the placenta [14];2) metabolites that accumulate when medication concentrations increase in the mother's body which can impact the fetus via inflammatory mediators [15], hormones [16], and toxins [17]; 3) deterioration of the maternal physical condition caused by the medication (repair or damage to several organs) which can affect the fetus; and 4) disruption of implantation due to medication effects [18].

The placenta, through which medications pass and are delivered to the fetus, also serves to filter the substances that pass through. The placenta is a metabolically active tissue that responds to the maternal environment, including perturbations during pregnancy, by regulating the fetal supply of nutrients and oxygen and the secretion of hormones into the maternal and fetal circulation [19]. Importantly, there might be differences in the transplacental transfer of medications due to significant functional differences of the placenta between the first two months of pregnancy (histiotrophic nutrition) and later in pregnancy (hemotrophic nutrition) [20]. In some cases, inflammatory mediators secreted from the mother can cross the placenta and affect the fetus [21]. Inflammatory processes in mothers can cause premature birth [15] and fetal damage regardless of preterm birth [22]. One study investigating the effects of persistent inhalation of chemicals by mothers reported that birthweight decreased with increasing exposure to contaminants [23], which is in agreement with the results of the subgroup analysis based on fetal survival performed in the present study. Our findings suggest that both pregnant women and neonates in the present study experienced not only direct lung damage but also systemic adverse effects caused by HDs through inhalation.

The analysis of pregnant women with their fetuses categorized according to maternal survival revealed that survival was related to the total duration of HD use, especially the duration of pre pregnancy HD use. These results may be associated with not only the direct effects of HDs but also rapid worsening of symptoms due to the progression of damage to various maternal organs, especially the lungs, with continuous exposure. Longer duration of HD use was associated with decreased maternal-fetal survival, consistent with studies reporting that HD use exacerbates HDLI in a dose-dependent manner [9].

Regarding immune tolerance that occurs during pregnancy, according to the "evolutionary non-self model," the presence of fetal antigens at the maternal-fetal interface does not necessarily activate the immune system. "Danger" and related models assert that danger signals are not expressed as long as the fetus is growing and developing normally and that the immune system is not activated to become harmful to the fetus. In the present study, the subgroup analysis based on maternal survival revealed that the onset of clinical symptoms after childbirth was associated with survival. In addition, the subgroup analysis based on fetal survival indicated that chances of maternal survival increased as time from the 
onset of clinical symptoms to birth diminished. However, maternal mortality increased in cases where the symptoms occurred before childbirth, indicating that the receptor-mediated immune response is activated in the presence of infection to protect the mother. Eventually, the change in the immune status of the mother due to HD use means that exceeding the threshold for immune tolerance is recognized as a "danger" state, leading to the deployment of a rapid immune response [23].

In mothers exposed to hazardous chemical material, the fetus may not be implanted or the neonate might have a lower birth weight [18]. In the present study, we performed subgroup analysis to determine the effect of HD exposure on trimester-specific survival rates. To this end, we grouped prepregnancy with the first trimester and the second trimester with the third trimester to evaluate exposure duration because the first trimester of pregnancy is considered to be a vulnerable period for implantation and fetal organ formation. In the first trimester, various issues, including abnormalities in the endocrine system, can have a critical impact on fetal development after fetal exposure to hazardous substances [14]. However, in the current study cohort, there were no cases of exposure in the first or third trimester in the subgroup with fetal and maternal death; therefore, statistical analysis could not be conducted. It is possible that exposure might lead to implantation difficulties in the first trimester. Additionally, the symptoms of mothers appeared before the 27th week in the group with both fetal and maternal death; therefore, there was no HD use in the third trimester in this group.

In Korea, summer is humid whereas spring, autumn, and winter are dry. Thus, we determined the survival rates specifically in summer and non-summer seasons and found that the proportion of mothers who became pregnant was higher in the spring and summer when HD use began to decline than in autumn and winter when HD use began to increase. One possibility is that an implantation disruption may occur when pregnancy starts in the autumn and winter during which HD use is relatively higher [18].

The current study has several limitations. First, the exact concentration and capacity of HD use and total seasonal consumption could not be confirmed. The information on HD use was obtained from blinded medical records and retrospectively reviewed; therefore, data to estimate HD concentrations, such as room size, average humidifier usage period, season of use, ventilation status, could not be obtained. To compensate for this limitation, converted dates were used, such as the date from the start of each event, seasonal classification, and trimester of exposure during pregnancy. Second, while we aimed to use objective data from the collected information, we cannot rule out recall bias over time. Third, the specific link between the effects of the maternal condition, such as hypoxia, hormones, inflammatory factors, abnormalities in metabolic processes, on fetal growth are unknown. Fourth, it is difficult to confirm the long-term consequences of HD exposure during the fetal period and to determine whether HD exposure affects the future growth and development of neonates, even those with no abnormalities at birth. Fifth, in mothers who are exposed to HDs, it is necessary to consider the individual differences in maternal reactions. Sixth, regarding premature babies, the differences in medical technology and development over the past 10 years might have affected mortality. Finally, we were not able to compare pregnant women who used HD with age-matched non-pregnant women who used HD or with age-matched pregnant women who did not use HD because women with no clinical symptoms did not use medical services or

Page $15 / 19$ 
give specific information about their HD use. Because of this limitation, we analyzed the data as a nested case-control study including subjects who used HD during pregnancy.

Despite these limitations, the current study investigated the rarely considered relationship between maternal and fetal survival in response to HD exposure, specifically including data on fetal survival in response to maternal inhalation exposure to small amounts of chemically hazardous substances with definitive maternal adverse effects. HDs do not generally have hazardous effects in humans; a large population throughout Korea has been exposed to HDs for a long duration. The present study included patients with clear respiratory symptoms. Because of various ethical and moral concerns, it is difficult to determine the effect of HD use in pregnant women. As a major strength, this is the first study investigating the survival of neonates born to mothers who were exposed to HDs during pregnancy.

\section{Conclusions}

In the present study examining neonatal survival in pregnancies with HD exposure, we found that shorter HD use was associated with higher maternal survival rate and that shorter time from birth to symptom onset was associated with higher likelihood of maternal-fetal survival. We also found that the surviving neonates born to mothers who survived had lower birth weights and that the maternal symptoms started before childbirth in the group of mothers and fetuses who died.

\section{Abbreviations}

BMI, Body mass index; HD, Humidifier disinfectants; HDLI, HD lung injury; ILD, Interstitial lung disease; KEITI, Korea Environment Industry \& Technology Institute

\section{Declarations}

The authors declare that they have no known competing financial interests or personal relationships that could have appeared to influence the work reported in this paper.

Ethics approval and consent to participate: The study protocol was approved by the Institutional Review Board of Hallym University Dongtan Sacred Heart Hospital (IRB No. 2019-12-014-001), and written informed consent to participate in the study was obtained from the parents or their guardians at enrollment. All methods were performed in accordance with the relevant guidelines and regulations.

Consent for publication: A written informed consent to participate in the study was obtained from the parents or their guardians at enrollment.

Availability of data and materials: The datasets used and/or analyzed during the current study are available from the corresponding author on reasonable request.

Competing interests: The authors declare they have no actual or potential competing financial interests. 
Funding: This work was supported by the Environmental Health Center for Hazardous Chemical Exposure funded by the Ministry of Environment of the Republic of Korea (2017-2019).

Authors' contributions: SC conceptualized and designed the study, collected data, performed the statistical analysis, interpreted the data, drafted the initial manuscript and reviewed and revised the manuscript. JSC participated in the study design and interpretation of analyses and critically reviewed the manuscript. All authors reviewed and approved the final manuscript.

Acknowledgments: The authors would like to thank all study subjects for their cooperation in this study. In particular, we are thankful for the clinical examination results and comments provided by the Environmental Health Center for Hazardous Chemical Exposure at National Medical Center, funded by the Ministry of Environment, Republic of Korea.

\section{References}

1. Daftary AS, Deterding RR. Inhalational lung injury associated with humidifier "white dust." Pediatrics. 2011;127:e509-12.

2. Korea Centers for Disease Control and Prevention (KCDC). Hospital based case-control study on lung injury with unknown causes [in Korean]. Chungcheongbuk-do, Korea: KCDC;2011:825-828. Public Health Weekly Report.

3. Kim YH. Humidifier disinfectant: 78 deaths and still in progress. Iran J Public Health. 2013;42:113.

4. Yang HJ, Kim HJ, Yu J, Lee E, Jung YH, Kim HY, et al. Inhalation toxicity of humidifier disinfectants as a risk factor of children's interstitial lung disease in Korea: a case-control study. PLOS ONE. 2013;8:e64430.

5. Paek D, Koh Y, Park DU, Cheong HK, Do KH, Lim CM, et al. Nationwide study of humidifier disinfectant lung injury in South Korea, 1994-2011. Incidence and dose-response relationships. Ann Am Thorac Soc. 2015;12:1813-21.

6. Ryu SH, Park DU, Lee E, Park S, Lee SY, Jung S, et al. Humidifier disinfectant and use characteristics associated with lung injury in Korea. Indoor Air. 2019;29:735-47.

7. Jeon $\mathrm{BH}$, Park YJ. Frequency of humidifier and humidifier disinfectant usage in gyeonggi provine. Environ Health Toxicol. 2012;27:e2012002.

8. Park DU, Ryu SH, Roh HS, Lee E, Cho HJ, Yoon J, et al. Association of high-level humidifier disinfectant exposure with lung injury in preschool children. Sci Total Environ. 2018;616:855-62.

9. Chang MH, Park H, Ha M, Kim Y, Hong YC, Ha EH. Characteristics of humidifier use in Korean pregnant women: the mothers and children's environmental health (MOCEH) study. Environ Health Toxicol. 2012;27:e2012003.

10. Yoon J, Cho HJ, Lee E, Choi YJ, Kim YH, Lee JL, et al. Rate of humidifier and humidifier disinfectant usage in Korean children: a nationwide epidemiologic study. Environ Res. 2017;155:60-3. 
11. Statistics Korea. 2009-2011 Results of compensation for causes of death (infants, mothers, and perinatal deaths); 2012.

12. KEITI. Comprehensive portal for humidifier disinfectant damage support. South Korea: KEITI; 2020. https://www.healthrelief.or.kr/home/content/stats01/view.do. Accessed 09 Jul 2021.

13. Mallozzi M, Bordi G, Garo C, Caserta D. The effect of maternal exposure to endocrine disrupting chemicals on fetal and neonatal development: a review on the major concerns. Birth Defects Res $C$ Embryo Today. 2016;108:224-42.

14. Salminen A, Paananen R, Vuolteenaho R, Metsola J, Ojaniemi M, Autio-Harmainen $\mathrm{H}$, et al. Maternal endotoxin-induced preterm birth in mice: fetal responses in toll-like receptors, collectins, and cytokines. Pediatr Res. 2008;63:280-6.

15. Unüvar T, Büyükgebiz A. Fetal and neonatal endocrine disruptors. J Clin Res Pediatr Endocrinol. 2012;4:51-60.

16. Wang X, Wang W, Cheng G, Huang L, Chen D, Tao Y, et al. High risk of embryo-fetal toxicity: placental transfer of T-2 toxin and its major metabolite HT-2 toxin in BeWo cells. Toxicol Sci. 2014;137:168-78.

17. Shirangi A, Nieuwenhuijsen M, Vienneau D, Holman CD. Living near agricultural pesticide applications and the risk of adverse reproductive outcomes: a review of the literature. Paediatr Perinat Epidemiol. 2011;25:172-91.

18. Dimasuay KG, Boeuf P, Powell TL, Jansson T. Placental responses to changes in the maternal environment determine fetal growth. Front Physiol. 2016;7:12.

19. Koren G, Ornoy A. The role of the placenta in drug transport and fetal drug exposure. Expert Rev Clin Pharmacol. 2018;11:373-85.

20. Kohmura Y, Kirikae T, Kirikae F, Nakano M, Sato I. Lipopolysaccharide (LPS)-induced intra-uterine fetal death (IUFD) in mice is principally due to maternal cause but not fetal sensitivity to LPS. Microbiol Immunol. 2000;44:897-904.

21. Buhimschi IA, Buhimschi CS, Weiner CP. Protective effect of N-acetylcysteine against fetal death and preterm labor induced by maternal inflammation. Am J Obstet Gynecol. 2003;188:203-8.

22. Govarts E, Remy S, Bruckers L, Den Hond E, Sioen I, Nelen V, Baeyens W et al., Combined effects of prenatal exposures to environmental chemicals on birth weight. Int J Environ Res Public Health. 2016;13:495.

23. Bonney EA. Alternative theories: pregnancy and immune tolerance. J Reprod Immunol. 2017;123:6571.

\section{Figures}




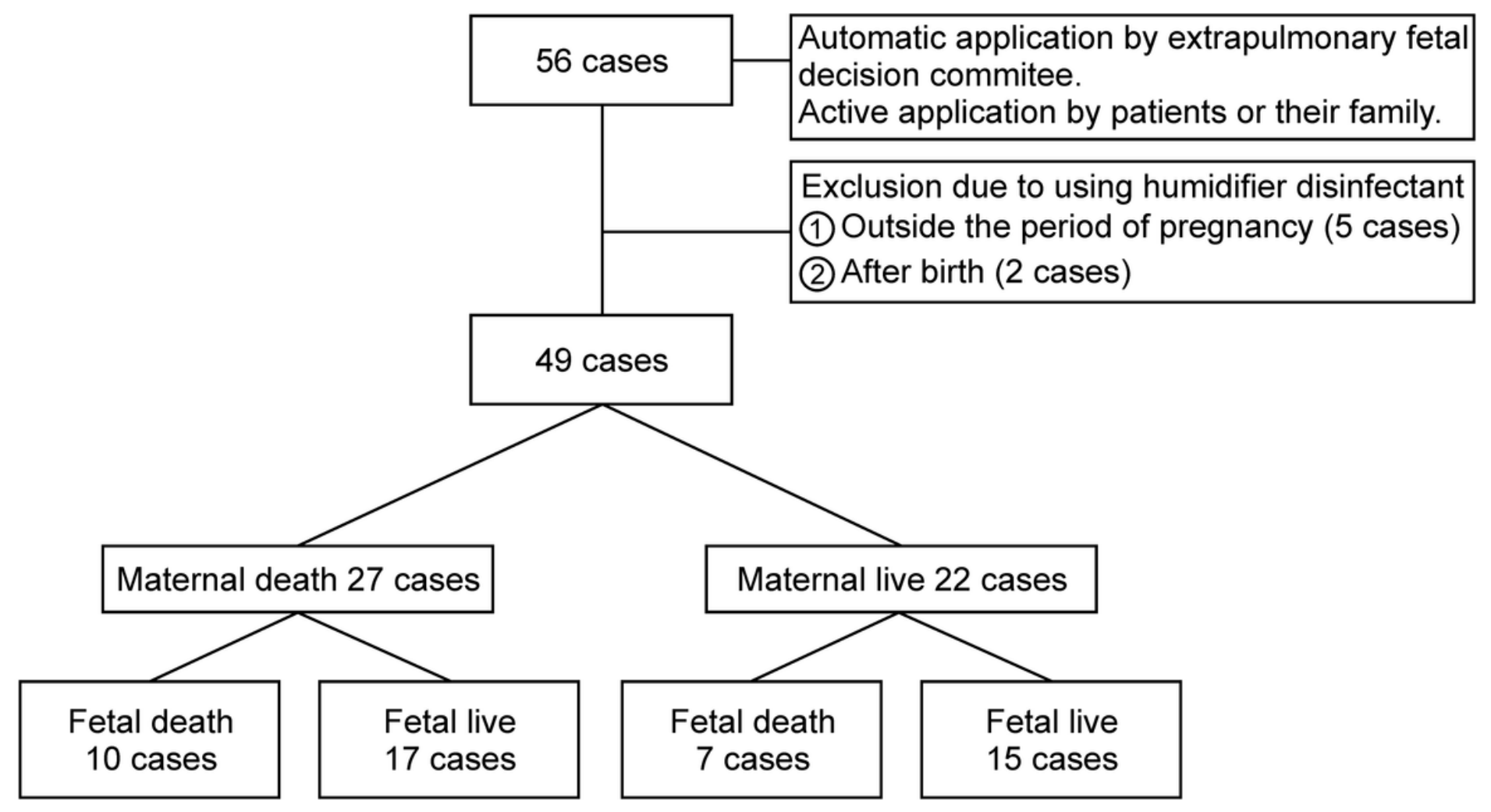

Figure 1

Study flowchart $(n=56)[2,13]$ 